\title{
Revisiting the Derivation of Heisenberg's Uncertainty Principle: The Collapse of Uncertainty at the Planck Scale*
}

\author{
Espen Gaarder Haug \\ Norwegian University of Life Sciences
}

September 4, 2018

\begin{abstract}
In this paper, we will revisit the derivation of Heisenberg's uncertainty principle. We will see how the Heisenberg principle collapses at the Planck scale by introducing a minor modification. The beauty of our suggested modification is that it does not change the main equations in quantum mechanics; it only gives them a Planck scale limit where uncertainty collapses. We suspect that Einstein could have been right after all, when he stated, "God does not throw dice." His now-famous saying was an expression of his skepticism towards the concept that quantum randomness could be the ruling force, even at the deepest levels of reality. Here we will explore the quantum realm with a fresh perspective, by re-deriving the Heisenberg principle in relation to the Planck scale.

We will show how this idea also leads to an upper boundary on uncertainty, in addition to the lower boundary. These upper and lower boundaries are identical for the Planck mass particle; in fact, they are zero, and this highlights the truly unique nature of the Planck mass particle. Further, there may be a close connection between light and the Planck mass particle: In our model, the standard relativistic energy momentum relation also seems to apply to light, while in modern physics light generally stands outside the standard relativistic momentum energy relation.

We will also suggest a new way to look at elementary particles, where mass and time are closely related, consistent with some of the recent work in experimental physics. Our model leads to a new time operator that does not appear to be in conflict with the Pauli objection. This indicates that both mass and momentum come in quanta, which are perfectly correlated to an internal Compton 'clock' frequency in elementary particles.

Key words: Heisenberg's uncertainty principle, certainty, wave function, Planck scale, Planck mass, Planck particle, Bell's Inequality, superposition, entropy, Compton clock.
\end{abstract}

\section{Introduction to the Momentum and Energy Operators}

A commonly used wave function ${ }^{1}$ in quantum mechanics is

$$
\Psi(x, t)=e^{i(k x-\omega t)}
$$

where $\omega=\frac{E}{\hbar}$, and

$$
k=\frac{2 \pi}{\lambda}
$$

From the de Broglie matter wave, we know that

$$
\lambda=\frac{h}{p}
$$

This means we have

$$
k=\frac{p}{\hbar}
$$

and this means we can write the wave equation also as (well-known)

$$
\Psi=e^{i\left(\frac{p}{\hbar} x-\frac{E}{\hbar} t\right)}
$$

*Thanks to Victoria Terces for helping me edit this manuscript. Thanks also to several anonymous reviewers for useful comments.

1 The plane wave solution to the Klein-Gordon equation and the Schrödinger equation. 
Next we take the partial derivative with respect to $x$ and get

Multiplying each side by $\frac{\hbar}{i}$ we get

$$
\frac{\partial \Psi}{\partial x}=\frac{i p}{\hbar} \Psi
$$

$$
\begin{aligned}
\frac{\hbar}{i} \frac{\partial \Psi}{\partial x} & =p \Psi \\
-i \hbar \frac{\partial \Psi}{\partial x} & =p \Psi
\end{aligned}
$$

and from this we have the well-known momentum operator

$$
\hat{p}=-i \hbar \frac{\partial}{\partial x}
$$

Next, we will use this information to derive Heisenberg's uncertainty principle.

\section{Introduction to Commutators, Operators, and Heisenberg's Uncertainty Principle}

A standard commutator is given by

$$
[\hat{A}, \hat{B}]=\hat{A} \hat{B}-\hat{B} \hat{A}
$$

If $[\hat{A}, \hat{B}] \neq 0$, then $\hat{A}$ and $\hat{B}$ do not commute. If $[\hat{A}, \hat{B}]=0$, then $\hat{A}$ and $\hat{B}$ do commute. Based on this, we have the following uncertainty

$$
\sigma_{A} \sigma_{B}=\frac{1}{2}|\langle\hat{A}, \hat{B}\rangle|=\frac{1}{2}\left|\int \Psi^{*}[\hat{A}, \hat{B}] \Psi d x\right|
$$

where $\Psi^{*}$ is the complex conjugate of $\Psi$, and we see from the expression above that if $\hat{A}$ and $\hat{B}$ commute, there is no uncertainty. The Heisenberg uncertainty principle $[1,2]$ can be derived from the following commutator

$$
[\hat{p}, \hat{x}]=\hat{p} \hat{x}-\hat{x} \hat{p}
$$

where the $\hat{p}$ is the momentum operator and $\hat{x}$ is the position operator. Again, the momentum operator is given by

and the position operator is given by

$$
\hat{p}=-i \hbar \frac{\partial}{\partial x}
$$

$$
\hat{x}=x
$$

From this we have

$$
\begin{aligned}
{[\hat{p}, \hat{x}] \Psi } & =[\hat{p} \hat{x}-\hat{x} \hat{p}] \Psi \\
& =\left(-i \hbar \frac{\partial}{\partial x}\right)(x) \Psi-(x)\left(-i \hbar \frac{\partial}{\partial x}\right) \Psi \\
& =-i \hbar\left(\Psi+x \frac{\partial \Psi}{\partial(x)}\right)+i \hbar x \frac{\partial \Psi}{\partial(x)} \\
& =-i \hbar\left(\Psi+x \frac{\partial \Psi}{\partial(x)}-\frac{\partial \Psi}{\partial(x)}\right) \\
& =-i \hbar \Psi
\end{aligned}
$$

And we have the following uncertainty

$$
\begin{aligned}
\sigma_{p} \sigma_{x} & \geq \frac{1}{2}\left|\int \Psi^{*}[\hat{p}, \hat{x}] \Psi d x\right| \\
& \geq \frac{1}{2}\left|\int \Psi^{*}(-i \hbar) \Psi d x\right| \\
& \geq \frac{1}{2}\left|-i \hbar \int \Psi^{*} \Psi d x\right|
\end{aligned}
$$


and since $\int \Psi^{*} \Psi d x$ must sum to 1 (there must be $100 \%$ probability for the particle to be somewhere), we are left with

$$
\begin{aligned}
\sigma_{p} \sigma_{x} & \geq \frac{1}{2}|-i \hbar| \\
\sigma_{p} \sigma_{x} & \geq \frac{\hbar}{2}
\end{aligned}
$$

that is as expected, we arrive at the Kennard version of Heisenberg's uncertainty principle. The Heisenberg uncertainty principle is the foundation of many of the results and interpretations of quantum mechanics.

\section{The Planck Scale and Haug's Maximum Velocity for Matter}

In 1899, Max Planck [3, 4] introduced what he called the 'natural units': the Planck mass, the Planck length, the Planck time, and the Planck energy. He derived these units using dimensional analysis, assuming that the Newton gravitational constant, the Planck constant, and the speed of light were the most important universal constants. Lloyd Motz, while working at the Rutherford Laboratory in 1962, [5-7] suggested that there was probably a very fundamental particle with a mass equal to the Planck mass that he called the "Uniton." Motz acknowledged that his Unitons (Planck mass particles) had far too much mass compared to known subatomic masses. He tried to address this issue by claiming that the Unitons had radiated most of their energy away:

According to this point of view, electrons and nucleons are the lowest bound states of two or more Unitons that have collapsed down to the appropriate dimensions gravitationally and radiated away most of their energy in the process. - Lloyd Motz

Others have suggested that there were plenty of Planck mass particles around just after the Big Bang; see [8], but that most of the mass of these super-heavy particles has radiated away. Modern physics has also explored the concept of a hypothetical Planck particle that has $\sqrt{\pi}$ more mass than the Uniton originally suggested by Motz. Some physicists, including Motz and Hawking, have suggested that such particles could be micro black holes [9-11]. Planck mass particles have even been proposed as candidates for cosmological dark matter, [12, 13].

We will suggest that the Planck mass particle only lasts for one Planck second and that its mass should be seen as approximately $1.17 \times 10^{-51} \mathrm{~kg}$ compared to other particles. This would give an alternative explanation of why we have not observed the Planck mass particle yet. Its lifetime is very short and instead of expecting a very large mass of $m_{p} \approx 2.17651 \times 10^{-8} \mathrm{~kg}$, we should look for the smallest mass there is: $m_{p} t_{p}=\frac{\hbar}{l_{p}} \frac{1}{c} \frac{l_{p}}{c}=\frac{\hbar}{c^{2}}$. The Planck mass particle is, in our view, the mass gap. It is an observational time window dependent mass. In other words, we do not need to link the missing Planck mass particle to the idea that it only existed at the beginning of the Big Bang, or in the form of dark matter or micro black holes, as others have proposed. Instead we will maintain that the Planck mass particle could be the building block of all elementary particles.

We suspect that all other masses are time-dependent as well, but this will likely first be noticeable when one is trying to measure their mass in an observational time window below their reduced Compton time, something we are not capable of doing at the moment. Recent experimental research [14] has concluded that "This directly demonstrates the connection between time and mass", where there is discussion about what the authors refer to as "Compton frequency."

We will come back to this idea repeatedly from a theoretical point of view in this article, in particular, under the section on time operators. The electron's mass can be found experimentally from the electron's reduced Compton wavelength [15]. To measure whether or not an electron mass is time-dependent, we would likely need an observational time interval of $\frac{\bar{\lambda}_{e}}{c}$, or less. That is to say, we suggest that elementary particles (internally) are a type of Compton clock. This supports our idea that the elementary particle mass could be dependent on the observed time interval, if that interval is below the reduced Compton time, $t_{c}=\frac{\bar{\lambda}}{c}$, of the particle in question

In a series of recent publications, Haug [16-19] has suggested that there is a maximum velocity for anything with rest-mass given by

$$
v_{\max }=c \sqrt{1-\frac{l_{p}^{2}}{\bar{\lambda}^{2}}}
$$

where $l_{p}$ is the Planck length. Further, $\bar{\lambda}$ is the reduced Compton wavelength of the elementary particle in question. The maximum velocity formula can be derived several ways: by assuming that the maximum relativistic mass an elementary particle can take is equal to the Planck mass, for example. An alternative derivation that gives the same result is to assume the shortest possible reduced Compton wavelength is the Planck length, even after relativistic length contraction, which gives 


$$
\begin{aligned}
\bar{\lambda} \sqrt{1-\frac{v^{2}}{c^{2}}} & \geq l_{p} \\
1-\frac{v^{2}}{c^{2}} & \geq \frac{l_{p}^{2}}{\bar{\lambda}^{2}} \\
v & \leq c \sqrt{1-\frac{l_{p}^{2}}{\bar{\lambda}^{2}}}
\end{aligned}
$$

Alternatively, we could have derived it from the assumption that the maximum frequency is the Planck frequency. One can also obtain the maximum velocity formula from the Heisenberg uncertainty principle by simply assuming that the smallest possible uncertainty in position is the Planck length; see Appendix A for a derivation. In addition, [16] contains a discussion of a precursor to this formula as well, but it had not been linked to the Planck length yet. The formula was first derived from atomism and shows how we get all the same mathematical end results as special relativity theory, but with this additional maximum velocity formula. This leads to Planck limits on a long series of formulas that otherwise have only infinity limits under standard physics. So, there are different ways to derive the formula that all seem to offer the same answer.

For any observed particle, the maximum velocity will be very close to that of the speed of light, but considerably above the speed achieved in the Large Hadron Collider. An electron has a reduced Compton wavelength of $\bar{\lambda}_{e} \approx 3.86159 \times 10^{-13} \mathrm{~m}$ and here we suggest that it can never be accelerated to a velocity faster than

$$
v=c \sqrt{1-\frac{l_{p}^{2}}{\bar{\lambda}_{e}^{2}}}=c \times 0.99999999999999999999999999999999999999999999912416
$$

In the above calculation, we have assumed a Planck length of $1.616199 \times 10^{-35} \mathrm{~m}$. As there is considerable uncertainty about the exact value for the Planck length, there is also some uncertainty about the theoretical value for the maximum speed limit of the electron. NIST (2014) CODATA reports a relative standard uncertainty for the Planck length of $2.3 \times 10^{-5}$ and a standard uncertainty of $0.000038 \times 10^{-35} \mathrm{~m}$. .

In our framework, the Planck length and the Planck mass can be measured independent of any prior knowledge of Newtonian gravity or the gravitational constant, as recently shown by $[19,20]$. Here we will show that the Heisenberg uncertainty principle breaks down at the Planck scale if the maximum velocity for matter follows this expression.

This also means there is a maximum limit on the relativistic momentum of

$$
P_{\max }=\frac{m v_{\max }}{\sqrt{1-\frac{v_{\max }^{2}}{c^{2}}}}=\frac{m c \sqrt{1-\frac{l_{p}^{2}}{\lambda^{2}}}}{\sqrt{1-\frac{\left(c \sqrt{1-\frac{l_{p}^{2}}{\lambda^{2}}}\right)^{2}}{c^{2}}}}=m_{p} c \sqrt{1-\frac{l_{p}^{2}}{\bar{\lambda}^{2}}}
$$

From the formula we see that the maximum momentum for most particles is very close to the Planck mass momentum $m_{p} c$. However, the Planck mass particle has zero momentum as $\bar{\lambda}=l_{p}$. We think that just as there is rest-mass energy, there is also what we can call "potential momentum," or rest-mass momentum, which is $m c$, but possibly only for the Planck mass particle. A momentum of $m c$ has often been related to massless particles, and we will soon come to that. The Planck mass particle is always at rest (when it exists, which is only for a Planck second), so it has potential momentum of $m_{p} c$, but zero traditional momentum in its rest state. No particle with rest-mass can move at the speed of light, and the fact that the Planck mass momentum is assumed to be $m_{p} c$ indicates it is not a normal momentum, but rather a rest-mass momentum.

Further, in the wave equation it is momentum and kinetic energy that are relevant. The maximum kinetic energy is given by 


$$
\begin{aligned}
E_{k} & =\frac{m c^{2}}{\sqrt{1-\frac{v_{m a x}^{2}}{c^{2}}}}-m c^{2} \\
& =\frac{m c^{2}}{\sqrt{1-\frac{\left(c \sqrt{1-\frac{l_{p}^{2}}{\lambda^{2}}}\right)^{2}}{c^{2}}}}-m c^{2} \\
& =\frac{m c^{2}}{\sqrt{1-1+\frac{l_{p}^{2}}{\lambda^{2}}}-m c^{2}} \\
& =m_{p} c^{2}-m c^{2} \\
& =\frac{\hbar}{\frac{1}{c} c^{2}-\frac{\hbar}{\bar{\lambda}} \frac{1}{c} c^{2}} \\
& =\hbar c\left(\frac{1}{l_{p}}-\frac{1}{\bar{\lambda}}\right)
\end{aligned}
$$

This means the wave function at the suggested maximum velocity for the anything with rest-mass is given by

$$
\begin{aligned}
\Psi & =e^{i\left(\frac{p_{\max }}{\hbar} x-\frac{E_{\max }}{\hbar} t\right)} \\
& =e^{i\left(\frac{m_{p} c \sqrt{1-\frac{l_{p}^{2}}{\lambda^{2}}}}{\hbar} x-\frac{\hbar c\left(\frac{1}{l_{p}}-\frac{1}{\lambda}\right)}{\hbar} t\right)}
\end{aligned}
$$

from this we have

$$
\begin{aligned}
\frac{\partial \Psi}{\partial x} & =\frac{i m_{p} c \sqrt{1-\frac{l_{p}^{2}}{\lambda^{2}}} \Psi}{\hbar} \Psi \\
\frac{\hbar}{i} \frac{\partial \Psi}{\partial x} & =m_{p} c \sqrt{1-\frac{l_{p}^{2}}{\overline{\lambda^{2}}} \Psi} \\
-i \hbar \frac{\partial \Psi}{\partial x} & =p \Psi
\end{aligned}
$$

so the momentum operator is

$$
\hat{p}=-i \hbar \frac{\partial}{\partial x}
$$

That is to say, the same momentum operator is just as before, so this will not change Heisenberg's uncertainty principle. However, there is one exception to the rule, namely for a Planck mass particle where the reduced Compton wavelength is $\bar{\lambda}=l_{p}$. Inserted into the wave equation, we get

$$
\begin{aligned}
\Psi & =e^{i\left(\frac{m_{p} c \sqrt{1-\frac{l_{p}^{2}}{l_{p}^{2}}}}{\hbar} x-\frac{\hbar c\left(\frac{1}{l_{p}}-\frac{1}{l_{p}}\right)}{\hbar} t\right)} \\
& =e^{i\left(\frac{m_{p} c \sqrt{1-1}}{\hbar} x-\frac{\hbar c(1-1)}{\hbar} t\right)} \\
& =e^{i\left(\frac{m_{p} c \times 0}{\hbar} x-\frac{\hbar c \times 0}{\hbar} t\right)}=1
\end{aligned}
$$

This means the wave function for the Planck mass particle in this view is always one. Bear in mind that $\Psi$ also is considered the probability amplitude, and that the probability amplitude squared is considered to be a probability. If we had gotten a negative value or a value above one, we should be concerned that our special case of the wave function was unphysical. The fact that we obtain one is, on the other hand, mathematically sound from a probability perspective as well. Still, what would the interpretation be if the Planck mass particle always has a probability of one? We think this should be seen in the light of the discussion of our maximum velocity formula. Namely that the Planck mass particle likely can only exist for one Planck second, and then it 
stands absolutely still. When we say the probability amplitude of the Planck mass particle always is one, that is naturally inside the time interval it exists, and it only exists for one Planck second.

This means that we have a momentum operator of

$$
\frac{\partial \Psi}{\partial x}=0
$$

Thus, the momentum operator must be zero for the Planck mass particle. Therefore, we must have

$$
\begin{aligned}
{[\hat{p}, \hat{x}] \Psi } & =[\hat{p} \hat{x}-\hat{x} \hat{p}] \Psi \\
& =\left(-0 \times \frac{\partial}{\partial x}\right)(x) \Psi-(x)\left(-0 \times \frac{\partial}{\partial x}\right) \Psi \\
& =0
\end{aligned}
$$

That is, $\hat{p}$ and $\hat{x}$ commute for the Planck particle, but do not commute for any other particle. For formality's sake, the uncertainty in the special case of the Planck particle must be

$$
\begin{aligned}
\sigma_{p} \sigma_{x} & \geq \frac{1}{2}\left|\int \Psi^{*}[\hat{p}, \hat{x}] \Psi d x\right| \\
& \geq \frac{1}{2}\left|\int \Psi^{*}(0) \Psi d x\right| \\
& \geq \frac{1}{2}\left|-0 \times \int \Psi^{*} \Psi d x\right|=0
\end{aligned}
$$

In the special case of the Planck mass particle, the uncertainty principle collapses to zero. In more technical terms this implies that the quantum state of a Planck mass particle can simultaneously be a position and a momentum eigenstate. That is, for the special case of the Planck mass particle we have certainty. In addition, the probability amplitude of the Planck mass particle will be one $\Psi_{p}=e^{0}=1$. However, we have claimed the Planck mass particle only lasts for one Planck second. We think the correct interpretation is that if one observes a Planck mass particle, then one automatically also knows its momentum, since a Planck mass particle (according to our maximum velocity formula) must stand still. In other words, for this, and only for this particle one knows the position and momentum at the same time. All particles other than the Planck mass particle will have a wide range of possible velocities for $v$, which leads to the uncertainty in the uncertainty principle.

We will claim the Planck mass particle always has rest-mass momentum equal to $m_{p} c$, and if we have detected a Planck mass particle, we know its position and its momentum. However, within one Planck second the Planck mass particle dissolves into energy. We also get a hint about the lifetime of a Planck particle from the Planck acceleration, $a_{p}=\frac{c^{2}}{l_{p}} \approx 5.56092 \times 10^{51} \mathrm{~m} / \mathrm{s}^{2}$. The Planck acceleration is assumed to be the maximum possible acceleration by several physicists; see [21, 22], for example. The velocity of a particle that undergoes Planck acceleration will actually reach the speed of light within one Planck second: $a_{p} t_{p}=\frac{c^{2}}{l_{p}} \frac{l_{p}}{c}=c$. However, we know that nothing with rest-mass can travel at the speed of light, so no "normal" particle can undergo Planck acceleration if the shortest possible acceleration time interval is the Planck second. The solution is simple. The Planck acceleration is an internal acceleration inside the Planck particle that within one Planck second turns the Planck mass particle into pure energy. This also explains why the Planck momentum is so special, namely always $m_{p} c$, unlike for any other particles, which can take a wide range of velocities and therefore a wide range of momentums.

In addition, for other elementary particles, such as electrons when accelerated to their maximum velocity $v_{\max }=c \sqrt{1-\frac{l_{p}^{2}}{\lambda^{2}}}$, we have

$$
\begin{aligned}
& \Psi=e^{i\left(\frac{p_{\max }}{\hbar} x-\frac{E_{\max }}{\hbar} t\right)} \\
& \Psi=e^{i\left(\frac{m_{p} c \sqrt{1-\frac{l_{p}^{2}}{\lambda^{2}}}}{\hbar} x-\frac{\hbar c\left(\frac{1}{l_{p}}-\frac{1}{\lambda}\right)}{\hbar} t\right)} \\
& \Psi=e^{i\left(\frac{\frac{\hbar}{l_{p}} \frac{1}{c} c \sqrt{1-\frac{l_{p}^{2}}{\lambda^{2}}}}{\hbar} x-c\left(\frac{1}{l_{p}}-\frac{1}{\lambda}\right) t\right)} \\
& \Psi=e^{i\left(\sqrt{\left.\frac{1}{l_{p}^{2}}-\frac{1}{\lambda^{2}} x-c\left(\frac{1}{l_{p}}-\frac{1}{\lambda}\right) t\right)}\right.}
\end{aligned}
$$


Assume now $x=l_{p}$ and $t=\frac{l_{p}}{c}$; we then get

$$
\begin{aligned}
& \Psi=e^{i\left(\sqrt{\frac{1}{l_{p}^{2}}-\frac{1}{\lambda^{2}}} l_{p}-c\left(\frac{1}{l_{p}}-\frac{1}{\lambda}\right) \frac{l_{p}}{c}\right)} \\
& \Psi=e^{i\left(\sqrt{\frac{l_{p}^{2}}{l_{p}^{2}}-\frac{l_{p}^{2}}{\bar{\lambda}^{2}}}-\left(\frac{l_{p}}{l_{p}}-\frac{l_{p}}{\lambda}\right)\right)} \\
& \Psi=e^{i\left(\sqrt{1-\frac{l_{p}^{2}}{\bar{\lambda}^{2}}}-1+\frac{l_{p}}{\lambda}\right)}
\end{aligned}
$$

and for any observed elementary particle so far, like the electron, $\bar{\lambda}>>l_{p}$ and since $1>>\frac{l_{p}^{2}}{\lambda^{2}}$ and $1>>\frac{l_{p}}{\lambda}$, this can be approximated very well as

$$
\Psi \approx e^{i(\sqrt{1}-1)}=e^{i \times 0}=1
$$

Again, we will claim the probability amplitude for any particle is one at the Planck scale, that is when observed at a Planck time interval when it moves at its maximum velocity. This means that the momentum and energy operators must be zero and uncertainty collapses at the Planck scale, when any elementary particle is accelerated to our suggested maximum velocity and where its relativistic mass is the Planck mass. This points towards the uniqueness of the Planck scale.

\subsection{Composite particles and maximum velocity}

Some may wonder about how our suggested maximum velocity behaves in relation to composite particles that consist of elementary particles with different reduced Compton wavelengths. The maximum velocity of any composite object (even a nucleus) is likely to be limited by the elementary particle with the shortest reduced Compton wavelength from which it is constructed, as first suggested by [18]. Our interpretation, based on extensive theoretical research, indicates that the elementary particle could burst into energy when reaching its maximum velocity. A composite particle such as a nucleus would then likely break apart. Interestingly, the energy levels we are talking about in relation to the maximum velocity are not far above the Hagedorn temperature. The Hagedorn temperature [23] is a theoretical temperature where quarks can be extracted (evaporated) from a proton. The Hagedorn temperature is extremely high; according to traditional grand-unified string models, it is said to be about $10^{30}$ kelvin, see [24]. The Hagedorn temperature is about two orders of magnitude smaller than the Planck temperature, which is $\frac{m_{p} c^{2}}{k_{b}} \approx 1.42 \times 10^{32}$ kelvin. In other words, it is not that strange to expect that the nucleus must break apart when the elementary particle with the shortest reduced Compton wavelength reaches its maximum velocity as suggested by the formula here, which leads to a Planck energy and therefore a Planck temperature in the elementary particle. This, however, indicates that the nucleus, if consisting of elementary particles with different reduced Compton wavelengths, would have a temperature somewhat below the Planck temperature when first breaking apart. As we are talking about quantum physics, the elementary particle reaching its maximum velocity will have a local temperature of exactly the Planck temperature at that point in time, but the composite particle must have a somewhat lower temperature. Whether or not this is somehow directly linked to the Hagedorn temperature we do not know at this point in time, but it could be worth further exploration.

\section{A New Time Operator That Does Not Seem To Be in Conflict with the Pauli Objection}

Time operators have not been commonly used in quantum mechanics. Time in quantum mechanics has, therefore, typically been considered only a parameter, but not an operator. The main resistance against time operators can be traced back to Wolfgang Pauli's strong objection [25] regarding the existence of a self-adjoint time operator. As we understand it, the Pauli objection is closely related to the concept that energy and time will not have the same spectrum.

Pauli's objections have encountered several counterexamples, criticisms, and discussions; see, for example, [26-40]. Some have taken the Pauli objection to the extreme, and argued that time between two events is meaningless in quantum mechanics, [41], "I prove that quantum theory rules out the possibility of any quantity that one might call 'the time interval between two events.". Others have tried to come up with creative, yet potential acceptable time operators by introducing dynamic time operators, or clocks that are outside the quantum system and therefore may be able to bypass the Pauli objection. Here we will suggest a new time operator. Modern physics, despite enormous progress in understanding time (in particular through the work of Larmor [42] and Einstein's special relativity theory [43]), does not have all the answers on what time is, or is 
not, at the deepest quantum level. In our view, elementary particles may be seen as subatomic quantum clocks. However, this new perspective does not mean we claim to have all the answers either and further work is to be done.

We will suggest a new way to look at particles that is related to Schrödinger's [44] hypothesis in 1930 of a ("trembling motion" in German) in the electron. Schrödinger indicated that the electron was in a sort of trembling motion $\frac{2 m c^{2}}{\hbar} \approx 1.55269 \times 10^{21}$ per second. We will suggest that the electron is in a Planck mass state $\frac{c}{\lambda_{e}} \approx 7.76344 \times 10^{20}$ times per second (exactly half of that of Schrödinger's "Zitterbewegung" frequency). However, each Planck mass state only lasts for one Planck second and we therefore get the normal electron mass from

$$
\frac{c}{\bar{\lambda}_{e}} m_{p} \frac{l_{p}}{c}=\frac{\hbar}{\bar{\lambda}_{e}} \frac{1}{c} \approx 9.10938 \times 10^{-31} \mathrm{~kg}
$$

We can also look at the same idea from a slightly different angle. It is well-known that the mass of any elementary particle can be expressed as

$$
m=\frac{\hbar}{\bar{\lambda}} \frac{1}{c}
$$

This can be rewritten as

$$
\begin{aligned}
m & =\frac{\hbar}{\bar{\lambda}} \frac{1}{c} \\
m & =\frac{\hbar}{\frac{c^{2}}{\bar{\lambda}}} \frac{1}{c} \\
m & =\frac{\hbar}{c^{2}} \frac{1}{\frac{\bar{\lambda}}{c}}
\end{aligned}
$$

The part $\frac{\bar{\lambda}}{c}$ we can call the reduced Compton time $t$, and we then have

$$
m=\frac{\hbar}{c^{2}} \frac{1}{t}
$$

again for example for an electron we have a reduced Compton time of $\frac{\bar{\lambda}}{c}=1.29 \times 10^{-21} \mathrm{~s}$, that again gives $m_{e}=\frac{\hbar}{c^{2}} \frac{1}{t} \approx 9.10938 \times 10^{-31} \mathrm{~kg}$.

That is to say, every elementary particle is also a clock ticking at the reduced Compton periodicity. The idea of an internal clock with a clock frequency close to the "Zitterbewegung" frequency in the case of the electron is not new; see [45, 46], for example. The link between mass and Compton time frequency seems to be supported by recent experimental research. Dolce and Perali [47] conclude that "the rest-mass of a particle is associated to a rest periodicity known as Compton periodicity". Again, in our model the reduced Compton periodicity is directly linked to a Planck mass event that lasts for one Planck second. In other words, we think the formula $m=\frac{\hbar}{c^{2}} \frac{1}{t}$ should be taken literally, that is all elementary particles ticking at the Compton periodicity $\frac{1}{t}$, where $t$ is the reduced Compton time, and that each tick is a Planck mass event lasting for one Planck second. This corresponds to the mass of known particles such as the electron, and we will show that it also leads to a sound time operator.

Be aware that $\frac{\hbar}{c^{2}}$ is indeed identical to the Planck mass times one Planck second. This also means that mass and momentum must be quantized and will be perfectly synchronized with the internal clock frequency of the elementary particle. This is not a surprise when each clock tick is a Planck mass lasting for one Planck second. The mass is quantized to the Planck mass times the Planck time per reduced Compton time and, in this model, the time-dependent momentum must be coming in quanta of

$$
p_{p}=m_{p} c
$$

In addition, the Planck momentum should be seen as a momentum only lasting for one Planck second. This means that even the velocity must come in quanta, and so we have

$$
\begin{aligned}
m v & \geq m_{p} c t_{p} \\
\overline{\bar{\lambda}} \frac{1}{c} v & \geq \frac{\hbar}{l_{p}} \frac{1}{c} c \frac{l_{p}}{c} \\
\frac{\hbar}{\bar{\lambda}} \frac{1}{c} v & \geq \frac{\hbar}{c} \\
v & \geq \bar{\lambda}_{e}
\end{aligned}
$$


Here we think the interpretation should be that in a given observational time window, the elementary particle must have moved at least its own reduced Compton wavelength for us to measure any momentum, even theoretically. In other words, this does not necessarily mean that there is a minimum velocity for elementary particles, but rather that a given elementary particle must be observed in a time window long enough to observe the particle move one Compton wavelength in order for us to discuss the momentum of that particle.

Turning to the plane wave function of the Klein-Gordon equation, this can be written as

$$
\Psi=e^{\frac{i}{\hbar}(p x-E t)}
$$

Replacing the momentum $p$ and the energy $E$, with their relativistic formulas gives

$$
\Psi=e^{\frac{i}{\hbar}}\left(\frac{m v}{\sqrt{1-\frac{v^{2}}{c^{2}}}} x-\left(\frac{m c^{2}}{\sqrt{1-\frac{v^{2}}{c^{2}}}}-m c^{2}\right) t\right)
$$

One could easily be criticized for throwing something that looks like standard relativistic physics into the wave function. Therefore, it is important to realize that we are not allowing any mass here; this is not classical physics. We will claim that $m$ in the formula above (when used in the wave function) must be that of an elementary particle, and possibly the aggregates of elementary particles, as long as they are less than or equal to a Planck mass. Remember $m=\frac{\hbar}{\lambda} \frac{1}{c}=\frac{\hbar}{c^{2}} \frac{1}{t}$, and replacing this as the mass in the wave equation gives

$$
\begin{aligned}
& \Psi=e^{\frac{i}{\hbar}\left(\frac{\frac{\hbar}{c^{2}} \frac{1}{t} v}{\sqrt{1-\frac{v^{2}}{c^{2}}}} x-\frac{\frac{\hbar}{c^{2}} \frac{1}{t} c^{2}}{\sqrt{1-\frac{v^{2}}{c^{2}}}} t+\frac{\hbar}{c^{2}} \frac{1}{t} c^{2} t\right)} \\
& \Psi=e^{\frac{i}{\hbar}\left(\frac{\frac{\hbar}{c^{2}} \frac{1}{t} v}{\sqrt{1-\frac{v^{2}}{c^{2}}}} x-\frac{\hbar}{\sqrt{1-\frac{v^{2}}{c^{2}}}}+\hbar\right)}
\end{aligned}
$$

Now, taking the partial derivative with respect to the plane wave function with respect to time we get

$$
\begin{aligned}
& \frac{\partial \Psi}{\partial t}=-\frac{i x}{\hbar t} \frac{\frac{\hbar}{c^{2}} \frac{1}{t} v}{\sqrt{1-\frac{v^{2}}{c^{2}}}} e^{\frac{i}{\hbar}\left(\frac{\frac{\hbar}{c^{2}} \frac{1}{t} v}{\sqrt{1-\frac{v^{2}}{c^{2}}}} x-\frac{\hbar}{\sqrt{1-\frac{v^{2}}{c^{2}}}}+\hbar\right)} \\
& \frac{\partial \Psi}{\partial t}=-\frac{i x}{\hbar t} \frac{\hbar \frac{\hbar}{c^{2}} \frac{1}{t} v}{\sqrt{1-\frac{v^{2}}{c^{2}}}} \Psi \\
& \frac{\partial \Psi}{\partial t}=-\frac{i x}{\hbar t} p \Psi
\end{aligned}
$$

remember $t=\frac{\bar{\lambda}}{c}$ and if we set $x$ equal to the reduced Compton wavelength, that is $x=\bar{\lambda}$, and we get

$$
i \frac{\hbar}{c} \frac{\partial \Psi}{\partial t}=p \Psi
$$

This means that the time momentum operator is

$$
\hat{p}=i \frac{\hbar}{c} \frac{\partial}{\partial t}
$$

and the time operator we suggest is simply $\hat{t}=t$. These two operators are both time operators: the momentum time operator and the time operator. Based on its construction, this time operator must be Hermitian and selfadjoint. In other words, the Pauli objection likely does not hold in this instance. What quantum mechanics seems to have been missing is that elementary particles are functions of time; they are quantum clocks that tick in every reduced Compton time period. Each tick is the Planck mass that lasts for one Planck second $m_{p} t_{p}=\frac{\hbar}{c^{2}}$.

Next we will check to see whether the momentum operator and time operator commute or not 


$$
\begin{aligned}
{[\hat{p}, \hat{t}] \Psi } & =[\hat{p} \hat{t}-\hat{t} \hat{p}] \Psi \\
& =\left(i \frac{\hbar}{c} \frac{\partial}{\partial t}\right)(t) \Psi-(t)\left(i \frac{\hbar}{c} \frac{\partial}{\partial t}\right) \Psi \\
& =i \frac{\hbar}{c}\left(\Psi+t \frac{\partial \Psi}{\partial(t)}\right)-i \hbar t \frac{\partial \Psi}{\partial(t)} \\
& =i \frac{\hbar}{c}\left(\Psi+t \frac{\partial \Psi}{\partial(t)}-\frac{\partial \Psi}{\partial(t)}\right) \\
& =i \frac{\hbar}{c} \Psi
\end{aligned}
$$

As we can see they do not commute. Further, we get the following uncertainty relation

$$
\begin{aligned}
\sigma_{p} \sigma_{t} & \geq \frac{1}{2}\left|\int \Psi^{*}[\hat{p}, \hat{t}] \Psi d t\right| \\
& \geq \frac{1}{2}\left|\int \Psi^{*}\left(i \frac{\hbar}{c}\right) \Psi d t\right| \\
& \geq \frac{1}{2}\left|i \frac{\hbar}{c} \int \Psi^{*} \Psi d t\right|
\end{aligned}
$$

and since $\int \Psi^{*} \Psi d t$ must sum to 1 , we are left with

$$
\begin{aligned}
\sigma_{p} \sigma_{t} & \geq \frac{1}{2}\left|i \frac{\hbar}{c}\right| \\
\sigma_{p} \sigma_{t} & \geq \frac{\hbar}{2} \frac{1}{c}
\end{aligned}
$$

That is, we have a new momentum time uncertainty principle in addition to the known momentum position uncertainty principle.

One may then wonder if we multiply each side with the speed of light $c$, will we obtain the energy time uncertainty relationship given by Heisenberg

$$
\begin{aligned}
\sigma_{p} c \sigma_{t} & \geq \frac{\hbar}{2} \frac{1}{c} c \\
\sigma_{E} \sigma_{t} & \geq \frac{\hbar}{2}
\end{aligned}
$$

For many years, researchers in the field have questioned if the energy time uncertainty principle is valid, as it has been assumed that one cannot have a time operator. Here we have suggested a new perspective on elementary particles that indicates they are clocks ticking discretely through each reduced Compton time interval. Each elementary particle is a Planck mass for one Planck second during every reduced Compton time period. This would mean the elementary particles actually have a time tick quantization that correlates perfectly with the energy spectra. We need no dynamic time operator or no external clocks to try to get around the Pauli objection; we simply do so by understanding that elementary particles can be seen as quantum clocks. This means energy must come in quanta of $f \hbar$, where $f=\frac{c}{\lambda}$ is the Compton frequency (well known). This also means mass comes in quanta of $m_{p} t_{p}=\frac{\hbar}{c^{2}}$, which is our suggested mass gap for a given observational time window.

Our analysis also leads to an interesting hypothesis. The Pauli objection could be based on wrong premises. It is assumed one can have Hermitian and self-adjoint momentum and position operators, as it is assumed that position and momentum are continuous, but that one cannot derive the energy time operator, as time is considered continuous and energy has been observed to be discrete. But here we indicate that in relation to time, energy, momentum and mass, all likely are quantized. That is mass, time intervals, momentum, and energy are all perfectly correlated in the Compton clock mass model, and this is the reason that momentum and position operators can be used. It is also why our time operators can be used. However, this should be done in a consistent way, as we have demonstrated here. Naturally this is only a hypothesis and further theoretical and observational investigation is recommended.

\section{$5 \quad$ Maximum Uncertainty in Addition to Minimum Uncertainty?}

Next let us look at the maximum kinetic energy multiplied by the relativistic reduced Compton time of the particle in question 


$$
\begin{aligned}
& E_{k} t=\left(\frac{m c^{2}}{\sqrt{1-\frac{v_{\max }^{2}}{c^{2}}}}-m c^{2}\right) \frac{\bar{\lambda}}{c} \sqrt{1-\frac{v_{\max }^{2}}{c^{2}}}
\end{aligned}
$$

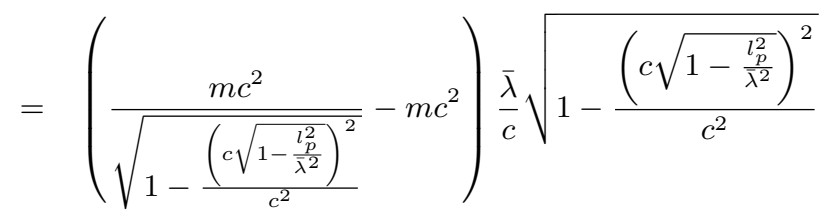

$$
\begin{aligned}
& =\left(\frac{m c^{2}}{\sqrt{1-1+\frac{l_{p}^{2}}{\bar{\lambda}^{2}}}}-m c^{2}\right) \frac{\bar{\lambda}}{c} \sqrt{1-1+\frac{l_{p}^{2}}{\bar{\lambda}^{2}}} \\
& =\left(m_{p} c^{2}-m c^{2}\right) \frac{l_{p}}{c} \\
& =\left(\frac{\hbar}{l_{p}} \frac{1}{c} c^{2}-\frac{\hbar}{\bar{\lambda}} \frac{1}{c} c^{2}\right) \frac{l_{p}}{c} \\
& =\hbar-\hbar \frac{l_{p}}{\bar{\lambda}} \\
& =\hbar\left(1-\frac{l_{p}}{\bar{\lambda}}\right)
\end{aligned}
$$

we will suggest that this is the maximum uncertainty for an elementary particle, so we must have

$$
\frac{\hbar}{2} \leq \sigma_{E} \sigma_{t} \leq \hbar\left(1-\frac{l_{p}}{\bar{\lambda}}\right)
$$

This means we have an extended uncertainty principle with lower boundary, similar to that of Heisenberg, as well as an additional new upper boundary. However, in the special case of a Planck mass particle, the lower and upper boundaries on uncertainty are zero. The correct interpretation here is that for the Planck mass particle we have a certainty principle. The energy times time for a Planck mass particle is always

$$
E_{p} t_{p}=m_{p} c^{2} \frac{l_{p}}{c}=\hbar
$$

Basically, this means if we detect a Planck mass particle we know it is at rest and it has a reduced Compton wavelength of $l_{p}$ that cannot undergo any length contraction, which is why it is at rest. The Planck mass particle can only have a rest-mass of $m_{p} c^{2}$ and a rest-mass momentum of $m_{p} c$, and must have zero momentum and zero kinetic energy. It stands absolutely still, but only for one Planck second before it dissolves into pure energy. This is also why its relativistic reduced Compton wavelength is certain, because its velocity is always zero (when it exists, but it only exists for one Planck second). Other particles have a velocity that can vary from zero to almost $c$; this means great uncertainty in their position, their relativistic reduced Compton wavelength, their relativistic mass, their relativistic momentum, and their relativistic kinetic energy. This interpretation is not the standard one, but we find it to be more logical.

The Planck mass particle, in our view, is also linked to photon-photon collisions. The velocity of a light particle at the precise moment when it collides with another light particle is the "melting point" of light and matter; see also [48]. With that we simply mean a photon likely must be considered matter at the instant it collides.

The uncertainty principle is, in this new perspective, actually an uncertainty about the velocity of the particle in question, that is in turn linked to the uncertainty in the relativistic reduced Compton wavelength of the particle. The uncertainty in the reduced Compton wavelength of a particle with momentum or kinetic energy different from zero must be

$$
\begin{array}{ccc}
l_{p} \geq & \bar{\lambda} \sqrt{1-\frac{(\Delta v)^{2}}{c^{2}}} & \leq \bar{\lambda} \\
l_{p} \geq & \Delta x & \\
\Delta x & & \leq \bar{\lambda}
\end{array}
$$

while for the Planck mass particle we have $\Delta \lambda=0$ because for the Planck mass particle it is always $\bar{\lambda}=l_{p}$, which must mean that the Planck mass particle cannot move; it is at absolute rest for one Planck second. 


\section{The Energy Momentum Relationship}

For a massless particle like a photon, a rational expectation would be to set the mass equal to zero in the energy momentum relationship. Then the energy momentum relationship gives zero energy, and therefore it is assumed not to hold for massless particles. In the case of massless particles, it is assumed that we simply have the following energy momentum relation $E=p c=\frac{c}{\lambda} h=f h$, where $f$ is the frequency. Here $E^{2}=p^{2} c^{2}+m^{2} c^{4}$ does not seems to apply for massless particles, something that is well-known. However, let us take a look at the standard momentum energy relation again. It can be written as

$$
\begin{aligned}
E^{2} & =p^{2} c^{2}+m^{2} c^{4} \\
E & =\sqrt{p^{2} c^{2}+\left(m c^{2}\right)^{2}} \\
E & =\sqrt{\left(\frac{m v}{\sqrt{1-\frac{v^{2}}{c^{2}}}}\right)^{2} c^{2}+\left(m c^{2}\right)^{2}} \\
E & =\sqrt{\frac{m^{2} c^{4}}{1-\frac{v^{2}}{c^{2}}}-m^{2} c^{4}+m^{2} c^{4}} \\
E & =\frac{m c^{2}}{\sqrt{1-\frac{v^{2}}{c^{2}}}}
\end{aligned}
$$

Bear in mind that the maximum velocity for a Planck mass particle is zero and that this gives

$$
\begin{aligned}
E & =\frac{m c^{2}}{\sqrt{1-\frac{v_{\max }^{2}}{c^{2}}}} \\
E & =m_{p}^{2} c^{2}=m_{p} c c=p_{p} c
\end{aligned}
$$

The Planck mass particle basically has the same energy momentum relationship as the massless particle: $E=p c$. However, massless particles are always expected to move at the velocity of light, while our maximum velocity for a Planck mass particle is the exact opposite, namely a velocity of zero. We do not disagree with the reasoning behind the speed of light, which is well-tested, but what is the speed of light exactly at the moment it deflects and changes direction, or when a particle is colliding with another light particle? Previously, we have suggested that the Planck mass particle likely is the collision point between two light particles [18], and it is not too controversial to claim that photon-to-photon collisions are linked to mass; see [48]. The energy $E=p_{p} c=m_{p} c c$ seems far too great for any observed photons. In our analysis, we claim that the Planck mass particle only lasts for one Planck second: $t_{p}=\frac{l_{p}}{c}$, before it dissolves into energy and once again travels at the speed of light. This means this means the minimum momentum of a photon is

$$
m_{p} c \frac{l_{p}}{c}=\frac{\hbar}{c}
$$

and multiplied by $c$ we get the energy of the light particle, that is $\hbar$, and dividing this by $c^{2}$ we get

$$
m=\frac{\hbar}{c^{2}}=m_{p} t_{p}
$$

We think the maximum velocity formula could be a key to understanding even the mass of massless particles.

\section{Short Discussion of Lorentz Symmetry}

Back to our break with Lorentz symmetry at the Planck scale. One could go on and compare our predicted Lorentz symmetry breakdown with other models that also predict Lorentz symmetry breakdown, including several quantum gravity models. However, as we are not yet able to test at such energy scales or to observe anything close to such time intervals (Planck time), we think such a comparison is better left to another time. Liberati [50] mentions several interesting constraints on Lorentz symmetry breaking. An important question is to what degree new physics at the Planck scale could be weakly detected at lower energies; this is discussed by $[51,52]$, for example. Initial investigation indicates that our maximum velocity formula leads to no detectable differences for known particles before one reaches velocities and energy levels far above what can be done at the Large Hadron Collider today. Still, there is room for further study, as a recent review article [53] on the possibility for Lorentz symmetry breaking in relation to quantum gravity predictions and experiments noted: 
In conclusion, though no violation of Lorentz symmetry has been observed so far, an incredible number of opportunities still exists for additional investigations.

In a wider perspective, we have suggested that all elementary particles are Compton clocks that tick at a reduced Compton frequency. In our model, each clock tick is a Planck mass lasting for one Planck second. This means the mass of elementary particles in our model will be observational time-dependent at time intervals below (or even close to) the reduced Compton time window. To our knowledge we have not been able to observe elementary particles in such short time intervals yet. However, recent observational research seems to support the idea that masses are linked to Compton time frequency [14, 47]. Further, we have indicated that the nucleus of an atom will likely break apart before it reaches the Planck temperature. Still, more research is clearly needed before any conclusion is made.

\section{Possible Implications}

Our maximum velocity of matter, which is directly linked to the Planck scale, has a series of potential implications for quantum mechanics that we will mention briefly here. These topics should be investigated further before a final conclusion is made.

\section{Renormalization}

Renormalization might no longer be needed. Even though renormalization has become an accepted method over time, this was not the case originally. One prominent critic of renormalization was Richard Feynman [54]. Clearly, he had a central role in the development of quantum electrodynamics, and yet he claimed

The shell game that we play ... is technically called 'renormalization'. But no matter how clever the word, it is still what I would call a dippy process! Having to resort to such hocus-pocus has prevented us from proving that the theory of quantum electrodynamics is mathematically self-consistent. It's surprising that the theory still hasn't been proved self-consistent one way or the other by now; I suspect that renormalization is not mathematically legitimate. - Richard Feynman, 1985

In 1987, Feynman [55] again commented on renormalization

Some twenty years ago one problem we theoretical physicists had was that if we combined the principles of quantum mechanics and those of relativity plus certain tacit assumptions, we seemed only able to produce theories (the quantum field theories), which gave infinity for the answer to certain questions. These infinities are kept in abeyance (and now possibly eliminated altogether) by the awkward process of renormalization. - Richard Feynman, 1987

Our maximum velocity limit provides a clear cut-off point on energy limits in elementary particles. Could this mean we no longer need renormalization?

\section{Bell's Theorem}

Several researchers have pointed out that by implicitly assuming all possible Bell measurements occur simultaneously, then all proofs of Bell's Theorem [56] violate Heisenberg's uncertainty principle [57]. What would it mean for the interpretation of Bell's Theorem if Heisenberg's uncertainty principle breaks down at the Planck scale and we then go from uncertainty to certainty (determinism)? Interestingly, Clover states [58]

By implicitly assuming that all measurements occur simultaneously, Bell's Theorem only applied to local theories that violated Heisenberg's uncertainty principle.

If Heisenberg's uncertainty principle breaks down at the Planck scale, this will likely open up the possibility of hidden variables, as suggested by Einstein, Podolsky, and Rosen in 1935; see [59]. We have shown that, under our theory, Planck mass particles can commute. Further, we claim that the Planck mass particle may be the building block of all other particles.

The three Pauli spin matrices operators (3-vector operators) are given by

$$
\begin{gathered}
S_{i}=\frac{\hbar}{2} \sigma_{i} \\
\hat{\sigma}_{x}=\left(\begin{array}{ll}
0 & 1 \\
1 & 0
\end{array}\right), \quad \hat{\sigma}_{y}=\left(\begin{array}{cc}
0 & -i \\
i & 0
\end{array}\right), \quad \hat{\sigma}_{z}=\left(\begin{array}{cc}
1 & 0 \\
0 & -1
\end{array}\right)
\end{gathered}
$$

If we can know the spin along two different axes at the same time, that would mean the spin commutators had to commute and that the commutator is equal to zero. From the Pauli matrices above, we can calculate the spin commutators, and we get the well-known result 


$$
\left[\hat{\sigma}_{x}, \hat{\sigma}_{y}\right]=2 i \hat{\sigma}_{z}, \quad\left[\hat{\sigma}_{y}, \sigma_{z}\right]=2 i \hat{\sigma}_{x}, \quad\left[\hat{\sigma}_{y}, \hat{\sigma}_{x}\right]=2 i \hat{\sigma}_{y}
$$

These are all significantly different from zero, which means they do not commute, and that we cannot measure two spin components simultaneously, according to the Pauli exclusion principle. However, since the momentum (and angular momentum) are always zero for a Planck mass particle, we think this indicates that the Planck mass particle must have zero spin. Thus, even if the Pauli matrices are non-relativistic, they should hold for the Planck mass particle. We note that the Pauli matrix operators were, to our knowledge, developed for particles with half spin; we will soon come back to how even half spin particles may be related to the Planck mass particle and zero spin.

Bear in mind that other elementary particles with half spin when reaching their maximum velocity, as defined by our formula, are closely related to the Planck mass particle, as they then have a relativistic mass equal to the Planck mass. As the maximum velocity for all known non-Planck mass particles is very close to the speed of light, an in-depth analysis would likely require a study of relativistic spin operators. However, it seems that at the Planck second, just after an elementary particle reaches it maximum velocity, it must stand still for a Planck second before bursting into energy. In addition, we will claim that an electron basically is a Planck mass particle in the order of about $10^{21}$ times a second (as measured from the same frame as electron rest frame); see discussion in the beginning of section 4. In our view it is "meaningless" to talk about a Planck mass particle outside an observational time window of one Planck second, as this is the life time of a Planck mass particle and we suspect that the Planck mass particle will dissolve into energy within one Planck second. Based on this new view we suggest that there must exist a special case of the Pauli matrix operators that suddenly changes to the matrices below when the elementary particle reaches the Planck scale:

$$
\hat{\sigma}_{x}=\left(\begin{array}{ll}
0 & 0 \\
0 & 0
\end{array}\right), \quad \hat{\sigma}_{y}=\left(\begin{array}{ll}
0 & 0 \\
0 & 0
\end{array}\right), \quad \hat{\sigma}_{z}=\left(\begin{array}{ll}
0 & 0 \\
0 & 0
\end{array}\right)
$$

In this special case, their commutators must be zero; that is, they commute for the Planck mass particle

$$
\left[\hat{\sigma}_{x}, \hat{\sigma}_{y}\right]=0, \quad\left[\hat{\sigma}_{y}, \sigma_{z}\right]=0, \quad\left[\hat{\sigma}_{y}, \hat{\sigma}_{x}\right]=0
$$

This again opens up for hidden variables and means the Bell's Inequality is questionable in light of this model. In our model, an electron possibly commutes $\frac{c}{\lambda_{e}} \approx 7.76 \times 10^{20}$ times a second. But only for one Planck second each time, so only in about $\frac{l_{p}}{\lambda_{e}} \approx 4.19 \times 10^{-23} \%$ of the observational time. In other words, it is challenging to detect. We could say the hidden variables may be hidden at the Planck scale, that is at the Planck time interval, which is linked to the Planck length (and the speed of light, as $t_{p}=\frac{l_{p}}{c}$ ). We feel this alternative perspective opens up a new avenue in quantum mechanics, perhaps one that is more logical than a theory where there is superluminal spooky action at distance. Instead, what if we can show that something special happens at the Planck scale? We believe the natural units first introduced by Max Planck could be the key to moving forward in quantum mechanics, and our way of incorporating them through our maximum velocity for anything with rest-mass seems to be a reasonable approach. The alternative is to hold on to the notion that Lorentz symmetry and also Heisenberg's uncertainty principle always hold, even at the Planck scale, and that there is nothing special with the natural units of Max Planck: the Planck mass, the Planck length, Planck time, and the Planck energy. We think this conception is a mistake, and that further investigation is warranted.

This means that Bell's Inequality possibly does not hold, and that there should be more discussion and theoretical work on the topic. Experimentally, it may be difficult to confirm or disconfirm at this time, as it likely would require Planck scale measurements, something we have not attained yet and perhaps will not attain in the future. Still, if two theories are consistent with experiments, one should consider which theory is the simplest from a logical point of view.

\section{Negative Probabilities and Negative Energy: A Possible New Interpretation}

In addition to a minimum uncertainty of $\sigma_{p} \sigma_{x} \geq \frac{\hbar}{2}$, there is a maximum uncertainty of

$$
\sigma_{E} \sigma_{t} \leq \hbar c\left(1-\frac{l_{p}}{\bar{\lambda}}\right)
$$

Assume that we now multiply both sides with minus one and we get

$$
-\sigma_{E} \sigma_{t} \geq-\hbar c\left(1-\frac{l_{p}}{\bar{\lambda}}\right)
$$

In other words, we are basically flipping the sign of the energy operator (and the momentum operator). We speculate that the theoretical negative energy one can mathematically obtain from the relativistic energy momentum relationship (when used in connection with the Klein-Gordon equation), for example, should be interpreted to mean that there is an upper limit on the relativistic energy level of elementary particles. However, this view requires further investigation. 
Negative quasi probabilities are typically related to negative energies, as first pointed by Dirac [60] (see also $[55,61,62])$

Thus the two undesirable things, negative energy and negative probability, always occur together. - Paul Dirac, 1942

Negative probabilities could be linked to negative uncertainty. Both negative probabilities and negative uncertainty cannot exist in the real world, which naturally is impossible, but mathematically could it simply mean we have flipped the sign of the inequality and that there is a maximum limit on uncertainty, in addition to a lower bound? Negative probabilities have been used in recent times in an attempt to explain the spooky action at distance in Bell's Theorem, [63]. We do not think that is the ultimate solution to the challenge, but it does give a hint about what might have been missing in quantum mechanics, namely an exact upper limit on energy for elementary particles, and thereby (in our theory) also a minimum distance (in terms of minimum reduced Compton wavelength for a particle) where uncertainty collapses. As we have discussed, in the special case of the Planck mass, the upper and lower bound are zero, and thus there is no uncertainty in that case. There are no negative probabilities per se, they are just an indication of an upper boundary condition on the maximum velocity for anything with rest-mass.

\section{Entropy}

It has been shown that Heisenberg's uncertainty principle and wave mechanics are closely linked to entropy [64]. In 1957, Hirschman [65] showed that the Heisenberg principle could be expressed as a lower bound on the sum of entropies; see also [66]. This likely indicates that a minimum entropy is a function of Heisenberg's uncertainty principle. Here we have shown that for the Planck mass particle there is no uncertainty, under our assumption of a new maximum velocity of particles with rest-mass. This could mean that entropy collapses at the Planck scale (for the Planck mass particle). This collapse of entropy would possibly only last for one Planck second.

When we are working with non-Planck mass particles, we have suggested there must be an upper limit on uncertainty equal to $\sigma_{E} \sigma_{t} \leq \hbar\left(1-\frac{l_{p}}{\lambda}\right)$ in addition to the Heisenberg lower limit. If this is true, then it would likely mean an upper bound on entropy. This would change our entire view on entropy and include the concept that entropy collapses at the Planck scale. At this time, this is purely speculative and we will leave it for another time and deeper examination to see how this could be linked to entropy, in both a mathematical and a logical manner.

\section{A New Type of Quantum Probabilities}

Here we will here suggest a new way to look at particles that is related to Schrödinger's [44] hypothesis in 1930 of a ("trembling motion" in German) in the electron. It is also linked to our Heisenberg derivation that shows a breakdown of uncertainty for Planck mass particles. Schrödinger indicated that the electron was in a sort of trembling motion $\frac{2 m c^{2}}{\hbar} \approx 1.55269 \times 10^{21}$ per second. We will suggest that the electron is in a Planck mass state $\frac{c}{\bar{\lambda}_{e}} \approx 7.76344 \times 10^{20}$ per second (about half of that of Schrödinger's "Zitterbewegung" frequency). However, each Planck mass state only lasts for one Planck second and we therefore get the normal electron mass from

$$
\frac{c}{\bar{\lambda}_{e}} m_{p} \frac{l_{p}}{c}=\frac{\hbar}{\bar{\lambda}_{e}} \frac{1}{c} \approx 9.10938 \times 10^{-31} \mathrm{~kg}
$$

The time it takes for the light to travel the reduced Compton wavelength. And the part $\frac{\hbar}{c^{2}}$ is equal to the Planck mass multiplied by the Planck time.

This model leads to a new probabilistic quantum probability theory that seems to be consistent with our uncertainty relationship as described above. We will claim that every elementary particle has a quantum probability of

$$
P=\frac{l_{p}}{\bar{\lambda}}
$$

which, in this model, is the probability of finding an elementary particle in its Planck mass state in a one Planck second observational time window. As only the Planck mass particle has reduced Compton wavelength equal to the Planck length, only the mass state of the Planck mass is certain if one finds a Planck mass particle. So, we clearly see the probability for elementary particles will vary between zero and one. For zero probability, we need an infinite reduced Compton wavelength. An electron, for example, will have a probability of $P \approx 4.185 \times 10^{-23}$ to be in a Planck mass state for any hypothetical observational time window of one Planck second. This means that every elementary particle can be expressed as

$$
m=m_{p} P=m_{p} \frac{l_{p}}{\bar{\lambda}}
$$


The formula that shows how an elementary particle can be expressed as a Planck mass multiplied by $\frac{l_{p}}{\lambda}$ is not new, it was possibly first suggested by Hoyle, Burbidge, and Narlikar in 1994; see [67]. What is new here is that we are interpreting $\frac{l_{p}}{\lambda}$ as a quantum probability for the particle to be in a Planck mass state, and that this probability is directly linked to the Planck length, in addition to the reduced Compton length of the particle in question.

Further, we will assume the reduced Compton wavelength must undergo standard length contraction as measured with Einstein-Poincaré synchronized clocks when the particle is moving relative to the observer. This will also affect the quantum probability for the particle to be in a Planck mass state. Every elementary particle must then have a relativistic quantum probability that is

$$
P=\frac{\frac{l_{p}}{\lambda}}{\sqrt{1-\frac{v^{2}}{c^{2}}}}=\frac{l_{p}}{\bar{\lambda} \sqrt{1-\frac{v^{2}}{c^{2}}}}
$$

Many will likely protest here, because if we only rely on combining this with Einstein's special relativity theory [43] it means we can get relativistic probabilities above unity and even close to infinite probabilities. This would be absurd and would not lead to a good theory. However, Haug's maximum velocity of matter comes into play, for simplicity we repeat it:

$$
v_{\max }=c \sqrt{1-\frac{l_{p}^{2}}{\bar{\lambda}^{2}}}
$$

More important is that at this maximum velocity for each particle, the quantum relativistic probability can take on a maximum value of

$$
\begin{aligned}
P & =\frac{l_{p}}{\bar{\lambda} \sqrt{1-\frac{v_{m a x}^{2}}{c^{2}}}} \\
P & =\frac{l_{p}}{\bar{\lambda} \sqrt{1-\frac{\left(c \sqrt{1-\frac{l_{p}^{2}}{\lambda^{2}}}\right)^{2}}{c^{2}}}} \\
P & =\frac{l_{p}}{\bar{\lambda} \sqrt{1-\frac{c^{2}\left(1-\frac{l_{p}^{2}}{\bar{\lambda}^{2}}\right)}{c^{2}}}} \\
P & =\frac{l_{p}}{\bar{\lambda} \sqrt{1-1+\frac{l_{p}^{2}}{\lambda^{2}}}} \\
P & =\frac{l_{p}}{\bar{\lambda}} \frac{\bar{\lambda}}{l_{p}}=1
\end{aligned}
$$

Thus, Haug's maximum velocity very elegantly leads to a maximum quantum probability of one. This means we get a boundary condition on the quantum probability for each elementary particle for each Planck second of

$$
\begin{aligned}
& \frac{l_{p}}{\bar{\lambda}} \leq P \leq \frac{l_{p}}{\bar{\lambda} \sqrt{1-\frac{v_{\max }^{2}}{c^{2}}}} \\
& \frac{l_{p}}{\bar{\lambda}} \leq P \leq 1
\end{aligned}
$$

Still, the relativistic quantum probability range will be different for each elementary particle. This means Einstein's relativistic mass formula for elementary particles can be seen as

$$
\frac{m}{\sqrt{1-\frac{v^{2}}{c^{2}}}}=\frac{\frac{\hbar}{\lambda} \frac{1}{c}}{\sqrt{1-\frac{v^{2}}{c^{2}}}}=\frac{\hbar}{\bar{\lambda} \sqrt{1-\frac{v^{2}}{c^{2}}}} \frac{1}{c}
$$

The maximum relativistic mass for any particle is the Planck mass multiplied by the maximum relativistic probability, which is one, and not surprisingly we get

$$
\text { Expected relativistic maximum mass electron }=m_{p} \frac{l_{p}}{\bar{\lambda}_{e} \sqrt{1-\frac{v_{\max }^{2}}{c^{2}}}}=m_{p} \times 1=m_{p}
$$


How should we interpret this? It means that at its maximum velocity any subatomic particle becomes a Planck mass, when relying on our new type of quantum probabilities. This also means that the original Heisenberg uncertainty principle collapses and becomes the certainty principle at the Planck scale. In addition, the Lorentz symmetry is broken at the Planck scale, but not before that.

The Planck mass particle is a particularly interesting case; its reduced Compton wavelength is $\bar{\lambda}=l_{p}$, which gives a probability range for the Planck mass particle of

$$
\begin{aligned}
\frac{l_{p}}{l_{p}} & \leq P_{p} \leq 1 \\
1 & \leq P_{p} \leq 1
\end{aligned}
$$

This can only be true if the Planck particle quantum probability is always $P_{p}=1$. This naturally means there is no uncertainty for the Planck mass particle, as we have shown when re-deriving the Heisenberg uncertainty principle. Our interpretation is that the Planck mass particle is the very collision point of the light particles making up each elementary particle.

Table 1 shows the standard relativistic mass as well as the probabilistic approach; they are consistent. Be aware that there must be a maximum velocity limit on anything with mass; this will be equal to Haug's maximum velocity.

\begin{tabular}{|c|c|c|}
\hline & Standard approach & $\begin{array}{c}\text { Probabilistic approach } \\
\text { observation in one Planck second }\end{array}$ \\
\hline Electron mass & $m=\frac{m_{e}}{\sqrt{1-\frac{v^{2}}{c^{2}}}}=\frac{\frac{h}{\lambda_{e}} \frac{1}{c}}{\sqrt{1-\frac{v^{2}}{c^{2}}}}$ & $E[m]=m_{p} P_{e}=m_{p} \frac{l_{p}}{\bar{\lambda}_{e} \sqrt{1-\frac{v^{2}}{c^{2}}}} \geq m_{e}$ \\
\hline Proton mass & $m=\frac{m_{\mathbf{P}}}{\sqrt{1-\frac{v^{2}}{c^{2}}}}=\frac{\frac{\hbar}{\lambda_{P}} \frac{1}{c}}{\sqrt{1-\frac{v^{2}}{c^{2}}}}$ & $E[m]=m_{p} P_{P}=m_{p} \frac{l_{p}}{\bar{\lambda}_{P} \sqrt{1-\frac{v^{2}}{c^{2}}} \geq m_{\mathbf{P}}}$ \\
\hline Planck mass particle & $m_{p}=\frac{m_{p}}{\sqrt{1-\frac{0^{2}}{c^{2}}}}=m_{p}$ & $E[m]=m_{p} P_{p}=m_{p} \frac{l_{p}}{l_{p} \sqrt{1-\frac{0^{2}}{c^{2}}}}=m_{p}$ \\
\hline
\end{tabular}

Table 1: This table shows the standard relativistic mass as well as the probabilistic approach. Be aware of the notation difference between the Planck mass $m_{p}$ and the proton rest-mass $m_{\mathbf{P}}$, and $E[m]$ stands for the expected mass.

Table 2 shows the relativistic mass when a particle is traveling at its maximum velocity. This will always correspond to a relativistic mass equal to the Planck mass, and a quantum probability of one. Be aware that the particle when reaching this velocity, which is above what can be achieved at LHC, likely will burst into energy within one Planck second. So, the certainty we predict can only last for one Planck second when we are dealing with single particles.

\begin{tabular}{|c|c|c|}
\hline & "Standard" approach & $\begin{array}{c}\text { Probabilistic approach } \\
\text { observation in one Planck second }\end{array}$ \\
\hline Electron mass & $m=\frac{m_{e}}{\sqrt{1-\frac{v_{m a x}^{2}}{c^{2}}}}=m_{p}$ & $E[m]=m_{p} P_{e}=m_{p} \frac{l_{p}}{\bar{\lambda}_{e} \sqrt{1-\frac{v_{m a x}^{2}}{c^{2}}}}=m_{p} \times 1=m_{p}$ \\
\hline Proton mass & $m=\frac{m_{\mathbf{P}}}{\sqrt{1-\frac{v_{m a x}^{2}}{c^{2}}}}=m_{p}$ & $E[m]=m_{p} P_{P}=m_{p} \frac{l_{p}}{\bar{\lambda}_{P} \sqrt{1-\frac{v_{m a x}^{2}}{c^{2}}}=m_{p} \times 1=m_{p}}$ \\
\hline Planck mass particle & $m_{p}=\frac{m_{p}}{\sqrt{1-\frac{v_{m a x}^{2}}{c^{2}}}}=m_{p}$ & $E[m]=m_{p} P_{p}=m_{p} \frac{l_{p}}{l_{p} \sqrt{1-\frac{v_{m a x}^{2}}{c^{2}}}}=m_{p} \times 1=m_{p}$ \\
\hline
\end{tabular}

Table 2: This table shows the standard relativistic mass as well as the probabilistic approach at the maximum velocity only. Be aware of the notation difference between the Planck mass $m_{p}$ and the proton rest-mass $m_{\mathbf{P}}$, and $E[m]$ stands for the expected mass.

\section{The Schwarzschild Radius, the Planck Scale, and Heisen- berg's Uncertainty Principle}

Several quantum gravity theories predict modifications of Heisenberg's uncertainty principle near the Planck scale: a so-called modified and Generalized Uncertainty Principle (GUP), see [68-72], for example. Our approach seems to lead to an extension of such theories that possibly opens up new avenues for quantum gravity. We 
will see that the Schwarzschild radius is closely related to the Compton periodicity in matter, and will evaluate how this is linked to the Planck scale and Heisenberg's uncertainty principle. From the Schwarzschild metric $[73,74]$ solution of the Einstein field equation [75], we get a radius today known as the Schwarzschild radius that mathematically is given by modern physics as

$$
r_{s}=\frac{2 G m}{c^{2}}
$$

Here we will claim the Schwarzschild radius is also equal to the Compton frequency, over one Planck second, of the mass in question multiplied by the Planck length

$$
\frac{r_{s}}{2}=\frac{G m}{c^{2}}=\frac{c}{\frac{\hbar}{m c}} t_{p} l_{p}=\frac{c}{\bar{\lambda}} t_{p} l_{p}=f t_{p} l_{p}=\frac{c}{\bar{\lambda}} \frac{l_{p}}{c} l_{p}=l_{p} \frac{l_{p}}{\bar{\lambda}}
$$

However, it will be "meaningless" with a Compton frequency of less than one per observational time window, either one observes minimum one event or no events. This means that the smallest mass with a real Schwarzschild radius, remembering that the observational time window is one Planck second, must be the Planck mass

$$
\frac{r_{s}}{2}=f t_{p} l_{p}=\frac{c}{l_{p}} \frac{l_{p}}{c} l_{p}=1 \times l_{p}
$$

which is consistent with standard theory, so it is not a big surprise. If the mass is smaller than the Planck mass, we still have

$$
\frac{r_{s}}{2}=\frac{G m}{c^{2}}=f t_{p} l_{p}=\frac{c}{\bar{\lambda}} \frac{l_{p}}{c} l_{p}=l_{p} \frac{l_{p}}{\bar{\lambda}}
$$

where for elementary particles $\bar{\lambda} \geq l_{p}$ and where $\frac{l_{p}}{\lambda}$, in our view, should be interpreted as a probability for a Planck event to occur, as discussed in section 9. That is to say, if the frequency is below one in a Planck second observational time window, then we are talking about the probability of one such event, which must be between zero and one. For example, we will claim that an electron has a Schwarzschild radius equal to twice the Planck length, since it is an elementary particle. However, it only has a Schwarzschild radius $\frac{c}{\lambda_{e}}$ times per second, and the Schwarzschild radius only lasts for one Planck second. The Schwarzschild radius is directly linked to the Planck mass event; see section 3. This means that for an observational time window equal to the Planck time, the expected half Schwarzschild radius for an electron is

$$
E\left[\frac{r_{s}}{2}\right]=l_{p} \frac{l_{p}}{\bar{\lambda}_{e}} \approx 6.76 \times 10^{-58} \mathrm{~m}
$$

where $\bar{\lambda}_{e}$ is the reduced Compton wavelength of the electron. This Schwarzschild radius is much smaller than the Planck length, but it is only an expectation. The expected value itself will never be observed (even in theoretical measurements), only the Planck length Schwarzschild radius exists at an elementary particle level. This is because our model assumes that the electron and other elementary particles are Planck masses lasting for one Planck second at the elementary particles Compton periodicity, as explained earlier in this paper. And again this seems consistent with recent experimental research. Based on this Compton periodicity model combined with our theory, most of the time the electron does not have a Schwarzschild radius, but $\frac{c}{\lambda_{e}}$ times per second it will have a half Schwarzschild radius equal to the Planck length, lasting for one Planck second each time. Still it has an expected Schwarzschild radius for a given time interval that is a function of the probability of observing the real Planck length Schwarzschild radius, which is different than the expectation.

For moving elementary particles, the Compton wavelength contracts as observed from the stationary frame; this means an elementary particle must have a half Schwarzschild radius of

$$
\frac{r_{s}}{2}=l_{p} \frac{l_{p}}{\bar{\lambda} \sqrt{1-\frac{v^{2}}{c^{2}}}}
$$

When we replace $v$ with our maximum velocity for matter, in order to look at the limit, we have a half Schwarzschild radius of

$$
\frac{r_{s}}{2}=l_{p} \frac{l_{p}}{\bar{\lambda} \sqrt{1-\frac{v_{\max }^{2}}{c^{2}}}}=l_{p} \frac{l_{p}}{\bar{\lambda} \sqrt{1-\frac{\left(c \sqrt{1-\frac{l_{p}^{2}}{\bar{\lambda}^{2}}}\right)^{2}}{c^{2}}}}=l_{p} \times 1=l_{p}
$$

This means that when an elementary particle reaches its maximum velocity, it has a half Schwarzschild radius equal to the Planck length, as observed from the stationary frame. This is because the reduced Compton wavelength of the particle is contracted to the Planck length at this maximum velocity and the relativistic mass is then the Planck mass. In addition, the Compton time has been contracted to the Planck time, as observed from the stationary frame and the reduced Compton length has contracted to the Planck length. This is consistent 
with the analysis already presented in this paper, and it means there is no uncertainty in the Schwarzschild radius of a particle when it is reaching its maximum velocity. The logic behind this is that the particle (Planck mass) then will only last for one Planck second, before bursting into energy. Further, we can see that the Schwarzschild radius is related to a probability of one at this maximum velocity. In standard physics, particles with mass less than the Planck mass have no Schwarzschild radius. Our theory predicts that the Schwarzschild radius is unique and important in relation to gravity, but that particles with mass less than the Planck mass have an expected Schwarzschild radius because they are Planck mass particles within every reduced Compton time interval.

This also means the uncertainty in the expected Schwarzschild radius for an elementary particle with mass size smaller than the Planck mass always must be

$$
\begin{aligned}
& l_{p} \frac{l_{p}}{\bar{\lambda} \sqrt{1-\frac{0^{2}}{c^{2}}}} \leq \quad \frac{r_{s}}{2} \leq l_{p} \frac{l_{p}}{\bar{\lambda} \sqrt{1-\frac{(\Delta v)^{2}}{c^{2}}}} \\
& l_{p} \frac{l_{p}}{\bar{\lambda} \sqrt{1}} \leq \frac{r_{s}}{2} \leq l_{p} \frac{l_{p}}{\bar{\lambda} \sqrt{1-\frac{\left(c \sqrt{1-\frac{l_{p}^{2}}{\lambda}}\right)^{2}}{c^{2}}}} \\
& l_{p} \frac{l_{p}}{\bar{\lambda}} \leq \frac{r_{s}}{2} \leq l_{p} \frac{l_{p}}{\bar{\lambda} \sqrt{1-\frac{\left(c \sqrt{1-\frac{l_{p}^{2}}{\lambda}}\right)^{2}}{c^{2}}}} \\
& \frac{l_{p}^{2}}{\bar{\lambda}} \leq \frac{r_{s}}{2} \leq l_{p}
\end{aligned}
$$

This is equal to the lower boundary on the space distortion given in GUP by Adler, namely $\frac{l_{p}^{2}}{\lambda}$. This means, as pointed out by [69], that we must have

$$
\frac{r_{s}}{2} \bar{\lambda} \geq l_{p}^{2}
$$

However, here we also have an upper boundary on the uncertainty in the half Schwarzschild radius of a elementary particle equal to the Planck length. And since it is an uncertain Schwarzschild radius, it is more correct to talk about the expected Schwarzschild radius for elementary particles. This also means the smallest Schwarzschild radius uncertainty for a non-Planck mass (before reaching its maximum velocity) must be

$$
\Delta r_{s} \geq 2 l_{p}
$$

This seems to be consistent with the predictions by Adler's [68] General Uncertainty Principle (GUP). However, that is before the elementary particle reach its maximum velocity. At the maximum velocity, the uncertainty collapses and we get

$$
r_{s}=2 l_{p}
$$

When the uncertainty collapses, at the very moment the particle reaches its maximum velocity, the Schwarzschild radius becomes equal to twice the Planck length. Again, this collapse of uncertainty only lasts for one Planck second. Our theory is a deeper and further extension of GUP that may help us towards a unified quantum gravity theory. This naturally requires considerably more investigation, but we think that more researchers should be made aware of this way of looking at extensions of GUP. We expect this view must lead to a modification in standard gravity theories to be compatible with the quantum scale.

Haug [76] has also recently shown that the Schwarzschild radius can be measured independent of any knowledge of the Newton's gravitational constant and even mass size. For example, we can find the Schwarzschild radius of the Earth simply by measuring the gravitational acceleration at the surface of Earth and then using the following formula

$$
r_{e}=\frac{g r^{2}}{c^{2}}
$$

where $r$ is the radius of the Earth, $g$ is the gravity acceleration, and $c$ is the well-known speed of light (and also gravity). Also, using a Cavendish apparatus we can measure the Schwarzschild radius of a ball without any prior knowledge of the mass size or Newton's gravitational constant.

We think the Schwarzschild radius is essential, as it contains the Compton frequency of the mass per Planck second, multiplied by the Planck length of the object. To extract the Planck length from it, we need to know something about the mass size, or alternatively the Compton frequency of the mass of interest. However, if we simply wish to find the Schwarzschild radius, then this is not needed.

Interestingly, in our analysis, half the Schwarzschild radius of one mass unit must be 


$$
\frac{r_{s}}{2}=\frac{G \times \text { One mass unit }}{c^{2}}=\frac{G}{c^{2}}
$$

If the mass unit is one $\mathrm{kg}$, we have a Schwarzschild radius for the mass unit of

$$
r_{s}=\frac{G \times 1 \mathrm{~kg}}{c^{2}} \approx 7.43 \times 10^{-28} \mathrm{~kg}
$$

Naturally, we will also have the same finding if we go from the Compton frequency of a $\mathrm{kg}$ and multiply by the Planck time, times the Planck length

$$
f t_{p} l_{p}=\frac{c}{\frac{\hbar}{m c}} t_{p} l_{p}=\frac{c}{\frac{\hbar}{1 \mathrm{~kg} \times c}} t_{p} l_{p}=\approx 7.43 \times 10^{-28} \mathrm{~kg}
$$

This supports our argument that we only need $G$ when we want to find the weight of a gravitational object. The gravitational constant is needed to standardize the Schwarzschild with respect to weight, which is only needed if we want to measure the weight of the gravitational object. We will also claim a mass with a calculated reduced Compton wavelength less than the Planck length must be a composite mass.

\section{New Perspective on Micro Black Holes}

Our mass gap connection to the Planck mass also gives a new perspective on micro black holes. For a long time, there has been speculation on the possible existence of micro black holes [9-11], but none have actually been detected thus far. They are expected to be approximately the size of a Planck mass, which is much larger than any observed particle. In our model, the mass gap is observational time dependent. If the observational time window is one second, the mass gap is only $m_{g}=\frac{\hbar}{l_{p}} \frac{1}{c} t_{p}=\frac{\hbar}{c^{2}} \approx 1.17 \times 10^{-51}$. However, the so-called micro black hole only lasts for one Planck second before bursting into energy again. The micro black hole is the mass gap. This means that what one should be searching for is an incredibly small mass (the mass gap), rather than the relatively large mass based on the traditional view of micro black holes. Still, the view that micro black holes have a Planck mass size is not wrong. However, it is only within a Planck time observational time window that the micro black hole will have the Planck mass.

As discussed previously, the link between Compton time and mass is currently missing in standard quantum mechanics. Thus it may be useful to explore this new and extended perspective. In time, it may be possible to develop predictions that are testable and could distinguish this theory from other GUP theories. The key here is to understand that mass comes in Compton frequencies, and that the smallest possible Compton frequency is one in an observational time window of the Planck time. Elementary particles are, in this view, Compton mass clocks, where each Compton tick is the Planck mass lasting for one Planck second.

In our view, a so-called micro black hole is a particle with an escape velocity equal to the speed of light. So, let us look at the escape velocity of the mass gap. In the Newtonian model, the escape velocity is given by the following equation solved with respect to $v$.

$$
\frac{1}{2} m v^{2}-G \frac{m M}{r}=0
$$

This gives the well-known escape velocity formula of

$$
v=\sqrt{\frac{2 G M}{r}}
$$

However, this only holds in a very weak gravitational field, as we have used the kinetic energy approximation $E_{k}=\frac{1}{2} m v^{2}$ that only holds when $v<<c$. For a Planck mass, we have no kinetic energy, see section 5 and, in particular, section 6 . The Planck mass only has rest-mass energy that within one Planck second converts to pure energy, that is light. In the special case for the Planck mass particle, we think the equation to solve, therefore, should be

$$
m_{p} c^{2}-G \frac{m_{p} m_{p}}{l_{p}}=0
$$

which gives

$$
c=\sqrt{\frac{G m_{p}}{l_{p}}}
$$


This is $\frac{1}{\sqrt{2}}$ lower than the standard physics escape velocity formula. So, this derivation may not seem to be correct at first, as the relativistic kinetic energy is $E_{k}=\frac{m c^{2}}{\sqrt{1-\frac{v^{2}}{c^{2}}}}-m c^{2}$ and not $m_{p} c^{2}$, but again the Planck mass particle has no kinetic energy, or rather all its rest-mass energy turns into energy within one Planck second. Be aware that general relativity theory predicts the same escape velocity formula for Planck masses as the Newton weak field approximation; see [77]. That GR is identical to the Newton weak field approximation for escape velocity is a bit strange. For a strong field, we must have relativistic kinetic energy, but again in our maximum velocity formula formulation, the Planck mass has no kinetic energy and the Planck mass particle is very unique. This is why we get an escape velocity formula slightly different for the Planck mass particle. At the Planck scale, it looks like our model gives a different prediction than Newton and GR, something that should be investigated further. In the special case for the Planck mass particle, we will claim there also is no orbital velocity. It is not possible to orbit a gravitational field that makes you move at velocity $c$. As mentioned earlier in this paper, one attains the velocity by staying in the Planck mass gravitational field for one Planck second and the Planck mass acceleration field also only lasts for one Planck second. Our interpretation of so-called micro black holes is simply is that they are the mass gap, which is where energy becomes the Planck mass for one Planck second, before bursting into energy again and then moving on at the speed of light.

\section{Conclusion}

Based on Haug's recently suggested maximum velocity for matter, we have shown that the momentum and position operators, as well as the energy and time operators, commute at the Planck scale, but not before that. This means that Einstein may have been right, as it opens up the possibility for hidden variable techniques, and also means that Bell's Inequality does not necessarily hold. Further, this means that we get a relativistic quantum mechanics, where there should no longer be a need for renormalization, as we get an exact upper limit on energies linked to the Planck scale. Our new theory seems to be consistent in all aspects. It means that Lorentz symmetry is broken at the Planck scale, but not before that, something that a series of quantum gravity theories have predicted could be the case.

It is important to note that our modified quantum theory does not conflict with any common experiments in quantum mechanics. Our theory simply gives a new and we would say a more logical interpretation. We have also suggested a new way to look at the mass of elementary particles that indicates the difference between mass and time is not clear cut. This view leads to a time operator that does not seem to be in conflict with the Pauli objection.

Hopefully, future research efforts will design experiments that can distinguish between this theory and other theories concerning quantum probabilities and their interpretation.

\section{Appendix A}

Here we show that it is possible to back out the maximum velocity for elementary particles with rest-mass from Heisenberg's uncertainty principle simply by assuming the minimum uncertainty is the Planck length. If we assume that we know the rest-mass of the particle in question, an electron, for example, then the uncertainty in momentum must come from the uncertainty in the velocity. This means we have

$$
\begin{aligned}
\Delta p \Delta x & \geq \hbar \\
\frac{m \Delta v}{\sqrt{1-\frac{(\Delta v)^{2}}{c^{2}}}} & \geq \frac{\hbar}{\Delta x}
\end{aligned}
$$

Now if we set $\Delta x$ to what we know is the minimum possible uncertainty in it, namely the Planck length, and we know the rest-mass of the particle, then it is even more clear that what is causing the uncertainty in the momentum is the uncertainty in the velocity: 


$$
\begin{gathered}
\frac{m \Delta v}{\sqrt{1-\frac{(\Delta v)^{2}}{c^{2}}}} l_{p} \geq \hbar \\
\frac{\frac{\hbar}{\lambda} \frac{1}{c} \Delta v}{\sqrt{1-\frac{(\Delta v)^{2}}{c^{2}}}} l_{p} \geq \hbar \\
\frac{\frac{1}{c} \Delta v}{\sqrt{1-\frac{(\Delta v)^{2}}{c^{2}}}} \geq \frac{\bar{\lambda}}{l_{p}} \\
\frac{\Delta v}{\sqrt{1-\frac{(\Delta v)^{2}}{c^{2}}}} \geq \frac{\bar{\lambda}}{l_{p}}
\end{gathered}
$$

Solved with respect to $\Delta v$ this gives

$$
\begin{aligned}
\frac{v^{2}}{1-\frac{(\Delta v)^{2}}{c^{2}}} & \geq \frac{\bar{\lambda}^{2}}{l_{p}^{2}} c^{2} \\
(\Delta v)^{2} & \leq \frac{\bar{\lambda}^{2}}{l_{p}^{2}} c^{2}\left(1-\frac{(\Delta v)^{2}}{c^{2}}\right) \\
(\Delta v)^{2}\left(1+\frac{\bar{\lambda}^{2}}{l_{p}^{2}}\right) & \leq \frac{\bar{\lambda}^{2}}{l_{p}^{2}} c^{2} \\
(\Delta v)^{2} & \leq \frac{\frac{\bar{\lambda}^{2}}{l_{p}^{2}} c^{2}}{\left(1+\frac{\bar{\lambda}^{2}}{l_{p}^{2}}\right)} \\
\Delta v & \leq \frac{c}{\sqrt{1+\frac{l_{p}^{2}}{\bar{\lambda}^{2}}}}
\end{aligned}
$$

This is the maximum uncertainty in velocity for a subatomic particle with known mass or known reduced Compton wavelength. A Taylor series expansion gives

$$
v \leq \frac{c}{\sqrt{1+\frac{l_{p}^{2}}{\lambda^{2}}}} \approx c\left(1-\frac{1}{2} \frac{l_{p}^{2}}{\bar{\lambda}^{2}}+\frac{3}{8} \frac{l_{p}^{4}}{\bar{\lambda}^{4}}-\frac{5}{16} \frac{l_{p}^{6}}{\bar{\lambda}^{6}} \ldots\right)
$$

and the maximum velocity formula suggested by Haug is

$$
v_{\max }=c \sqrt{1-\frac{l_{p}^{2}}{\bar{\lambda}^{2}}} \approx c\left(1-\frac{1}{2} \frac{l_{p}^{2}}{\bar{\lambda}^{2}}+\frac{1}{8} \frac{l_{p}^{4}}{\overline{\lambda^{4}}}-\frac{1}{16} \frac{l_{p}^{6}}{\overline{\lambda^{6}}} \ldots\right)
$$

In both formulas we get a highly accurate result by using the first term of the Taylor series expansion (as long as it is not a Planck mass particle) and we see they are practically the same: one is just derived from special relativity theory, while also suggesting that the maximum relativistic mass is the Planck mass, which is equivalent to assuming the shortest possible reduced Compton wavelength is $l_{p}$.

Using the Kennard version of the Heisenberg uncertainty principle instead of Heisenberg's original form would mean a slightly different maximum velocity, namely

$$
v \leq \frac{c}{\sqrt{1+4 \frac{l_{p}^{2}}{\lambda^{2}}}}
$$

That is to say, for any particle with $\bar{\lambda}>>l_{p}$, we can approximate this very well with the first term of a Taylor series expansion

$$
v \leq c\left(1-\frac{2 l_{p}^{2}}{\bar{\lambda}^{2}}\right)
$$

This is basically same formula that Haug first suggested as the maximum velocity of matter, which was derived by assuming there existed an indivisible mass-less particle that was the building block of all matter. This was first presented in 2014 and was given as $v_{\max }=\frac{\bar{\lambda}^{2}-l_{p}^{2}}{\bar{\lambda}^{2}-l_{p}^{2}} \approx c\left(1-\frac{2 l_{p}^{2}}{\bar{\lambda}^{2}}\right)$. Bear in mind that the Heisenberg uncertainty principle in reality only holds for non-Planck mass particles, e.g, only for particles with a reduced Compton wavelength longer than the Planck length. We speculate that the reduced Compton wavelength also must come 
in quanta $\bar{\lambda}=Z \times l_{p}$, where $Z$ is a positive integer. That means that the minimum reduced Compton wavelength in relation to the Heisenberg uncertainty principle is $2 l_{p}$, for a particle with reduced Compton wavelength of $l_{p}$ we do not have uncertainty. Combining this with the Kennard version of the Heisenberg uncertainty principle we get a maximum velocity of uncertainty in elementary particles of

$$
\begin{aligned}
\frac{m \Delta v}{\sqrt{1-\frac{(\Delta v)^{2}}{c^{2}}}} 2 l_{p} & \geq \frac{\hbar}{2} \\
\frac{v^{2}}{1-\frac{(\Delta v)^{2}}{c^{2}}} & \geq \frac{2 \bar{\lambda}^{2}}{2 l_{p}^{2}} c^{2} \\
\Delta v & \leq \frac{c}{\sqrt{1+\frac{l_{p}^{2}}{\lambda^{2}}}}
\end{aligned}
$$

In other words, the maximum uncertainty in velocity seems to be directly linked to a maximum velocity formula that can be derived from special relativity theory, where one applies constraints, namely that the maximum relativistic mass of an elementary particle is the Planck mass. In the special case of $\bar{\lambda}=l_{p}$, the uncertainty in velocity becomes zero, which is consistent with our analysis in this paper.

\section{References}

[1] W. Heisenberg. Über den anschaulichen inhalt der quantentheoretischen kinematik und mechanik. Zeitschrift für Physik, (43):172-198, 1927.

[2] E. H. Kennard. Zur quantenmechanik einfacher bewegungstypen”. Zeitschrift für Physik, (44):326-352, 1927.

[3] M. Planck. Naturlische Masseinheiten. Der Königlich Preussischen Akademie Der Wissenschaften, p . 479., 1899.

[4] M. Planck. Vorlesungen über die Theorie der Wärmestrahlung. Leipzig: J.A. Barth, p. 163, see also the English translation "The Theory of Radiation" (1959) Dover, 1906.

[5] L. Motz. Gauge invariance and the structure of charged particles. Il Nuovo Cimento, 26(4), 1962.

[6] L. Motz. A gravitational theory of the mu meson and leptons in general. Rutherford Observatory, Columbia University, 1966.

[7] L. Motz. The quantization of mass. Rutherford Observatory, Columbia University, 1971.

[8] S. S. De. Quantum creation of highly massive particles in the very early universe. International Journal of Theoretical Physics, 2001.

[9] S. Hawking. Gravitationally collapsed objects of very low mass. Monthly Notices of the Royal Astronomical Society, 152, 1971.

[10] L. Motz and J. Epstein. The gravitational charge $1 / 2 \sqrt{\hbar c}$ as a unifying principle in physics. Il Nuovo Cimento, 51(1), 1979.

[11] G. M. Obermair. Primordial Planck mass black holes (ppmbhs) as candidates for dark matter? Journal of Physics, Conference Series, 442, 2013.

[12] J. H. Macgibbon. Can Planck-mass relics of evaporating black holes close the universe? Nature, $329,1987$.

[13] V. I. Dokuchaev and Y. N. Eroshenko. Black hole atom as a dark matter particle candidate. https://arxiv.org/abs/1403.1375, 2014.

[14] S. Lan, P. Kuan, B. Estey, D. English, J. M. Brown, M. A. Hohensee, and Müller. A clock directly linking time to a particle's mass. Science, 339, 2013.

[15] S. Prasannakumar, S. Krishnaveni, and T. K. Umesh. Determination of rest mass energy of the electron by a Compton scattering experiment. European Journal of Physics, 33(1), 2012.

[16] E. G. Haug. Unified Revolution: New Fundamental Physics. Oslo, E.G.H. Publishing, 2014.

[17] E. G. Haug. The gravitational constant and the Planck units. A simplification of the quantum realm. Physics Essays Vol 29, No 4, 2016. 
[18] E. G. Haug. The ultimate limits of the relativistic rocket equation. The Planck photon rocket. Acta Astronautica, , 136, 2017 (see also arXiv:1807.10280).

[19] E. G. Haug. Can the Planck length be found independent of big G ? Applied Physics Research, 9(6), 2017.

[20] E. G. Haug. Planck mass measured totally independent of big G utilising Mcculloch-Heisenberg Newtonian equivalent gravity. preprints.org, 2018.

[21] G. Scarpetta. Letter Nuovo Cimento,, 51, 1984.

[22] D. F. Falla and P. T. Landsberg. Black holes and limits on some physical quantities. European Journal of Physics, 15, 1994.

[23] T. Ericson and J. Rafelski. The tale of the Hagedorn temperature. CERN Courier, 2003.

[24] J. J. Atick and E. Witten. The Hagedorn transition and the number of degrees of freedom of string theory. Nuclear Physics B, 310(2), 1988.

[25] W. Pauli. Die allgemeinen prinzipien der wellenmechanik. Springer, Berlin, p.84, 190.

[26] J. Kijowski. On the time operator in quantum mechanics and the Heisenberg uncertainty relation for energy and time. Reports on Mathematical Physics, 6(3), 1974.

[27] V. S. Olkkovsky, E. Recami, and A. J. Gerasimchuk. Time operator in quantum mechanics. IL Nuovo Cimento, 22(2), 1974.

[28] P. Busch, M. Grabowski, and P. J. Lahtic. Time observables in quantum theory. Physics Letters A.

[29] M. Bauer. A time operator in quantum mechanics. Annals of Physics, 150, 1983.

[30] N. Grot, C. Rovelli, and R. S. Tate. Time of arrival in quantum mechanics. Phys. Rev. A, 54(6), 1996.

[31] J. Kijowski. Comment on the "arrival time" in quantum mechanics. Phys. Rev. A, 59, 1999.

[32] E. A. Galapon. Self-adjoint time operator is the rule for discrete semibounded Hamiltonians. Proceedings of the Royal Society of London A: Mathematical, Physical and Engineering Sciences, 458(2027), 2002.

[33] E. A. Galapon. Pauli's theorem and quantum canonical pairs: the consistency of a bounded, self-adjoint time operator canonically conjugate to a Hamiltonian with non-empty point spectrum. Philosophical Transactions of the Royal Society of London, 458(2018), 2002.

[34] L. Nanni. A new derivation of the time-dependent Schrödinger equation from wave and matrix mechanics. Advances in Physics Theories and Applications, 43, 2005.

[35] Z. Y. Wang and C. Xiong. How to introduce time operator. Annals of Physics, 322(10), 2007.

[36] M. Bauer. A dynamical time operator in Dirac's relativistic quantum mechanics. International Journal of Modern Physics A, 54(6), 2014.

[37] C. Kinyanjui and D. S. Wamalwa. On the existence of a non-relativistic Hermitian time operator. International Journal of Pure and Applied Mathematics, 110(3), 2016.

[38] S. Khorasani. Time operator in relativistic quantum mechanics. Communications in Theoretical Physics, $68(1), 2017$.

[39] M. Bauer. On the problem of time in quantum mechanics. European Journal of Physics, 38(3), 2017.

[40] J. Leon and L. Maccone. The Pauli objection. The Foundations of Physics, 47(12), 2017.

[41] H. Halvorsen. Does quantum theory kill time? Working paper Princeton University, 2010.

[42] J. J. Larmor. Aether and Matter: A Development of the Dynamical Relations of the Aether to Material Systems. Cambridge University Press, 1900.

[43] A. Einstein. On the electrodynamics of moving bodies. Annalen der Physik, English translation by George Barker Jeffery 1923, (17), 1905.

[44] E. Schrödinger. Über die kräftefreie bewegung in der relativistischen quantenmechanik. Sitzungsberichte der Preuischen Akademie der Wissenschaften. Physikalisch-mathematische Klasse, 1930. 
[45] M. Rivas. Measuring the internal clock of the electron. arXiv:0809.3635, 2008.

[46] M. Bauer. Electron channeling, de Broglie's clock and the relativistic time operator. arXiv:1403.4580, 2014.

[47] D. Dolce and Perali. On the Compton clock and the undulatory nature of particle mass in graphene systems. The European Physical Journal Plus, 130(41), 2015.

[48] O. J. Pike, F. Mackenroth, E. G. Hill, and S. J. Rose. A photon-photon collider in a vacuum Hohlraum. Nature Photonics, 8, 2014.

[49] F. Kislat and H. Krawczynski. Planck-scale constraints on anisotropic Lorentz and CPT invariance violations from optical polarization measurements. Physical Review D, 95, 2017.

[50] S. Liberati. Lorentz symmetry breaking: phenomenology and constraints. Journal of Physics: Conference Series, 631, 2005.

[51] G. Amelino-Cameliaa, B. J. Ellisc, N. E. Mavromatosa, D. V. Nanopoulos, and S. Sarkar. Potential sensitivity of gamma-ray burster observations to wave dispersion in vacuo. Nature, 393, 2998.

[52] C. M. Reyesa, S. Ossandonb, and C. Reyesc. Higher-order Lorentz-invariance violation, quantum gravity, and fine-tuning. Physics Letters B, 746, 2005.

[53] A. Hees and et al. Tests of Lorentz symmetry in the gravitational sector. Universe, 2(4), 2017.

[54] R. P. Feynman. QED: The Strange Theory of Light and Matter. Penguin, p. 128, 1985.

[55] R. P. Feynman. Negative probability. First published in the book Quantum Implications: Essays in Honour of David Bohm, by F. David Peat (Editor), Basil Hiley (Editor) Routledge 6 Kegan Paul Ltd, London E6 New York, pages 235-248, http://kh.bu.edu/qcl/pdf/feynmanr19850a6e6862.pdf, 1987.

[56] J. S. Bell. On the Einstein Podolsky Rosen paradox. Physics, 1, 1964.

[57] M. Clover. Bell's theorem: A critique. arXiv:quant-ph/0502016, 2005.

[58] M. Clover. Bell's theorem: A new derivation that preserves Heisenberg and locality. arXiv:quantph/0409058, 2005.

[59] A. Einstein, B. Podolsky, and N. Rosen. Can quantum mechanical description of physical reality be considered complete? Phys. Rev., (47):777-780, 1935.

[60] P. Dirac. The physical interpretation of quantum mechanics. Proc. Roy. Soc. London, (A 180):1-39, 1942.

[61] E. P. Wigner. On the quantum correction for thermodynamic equilibrium. Phys. Rev., 40, 1932.

[62] W. Pauli. Remarks on problem connected with the renormalization of quantized field. Il Nuovo Cimento, 4, 1956.

[63] Ai Yu. Khrennikov. Bell's inequality: Physics meets probability. arXiv:0709.3909, 2007.

[64] I. Bialynicki-Birula and J. Mycielski. Uncertainty relations for information entropy in wave mechanics. Communications in Mathematical Physics, 44(2), 1975.

[65] R. Hirschman. A note on entropy. American Journal of Mathematics, 79, 1957.

[66] R. Leipnik. Entropy and the uncertainty principle. Information and Control, 2, 1959.

[67] F. Hoyle, G. Burbidge, and J.V. Narlikar. The basic theory underlying the quasi-steady state cosmology. Working paper, Inter-University Center for Astronomy and Astrophysics, 1994.

[68] R. J. Adler and I. Santiago. On gravity and the uncertainty principle. Modern Physics Letters A, 14(20), 1999.

[69] R. J. Adler. Six easy roads to the Planck scale. American Journal of Physics, 78(9), 2010.

[70] F. Ahmadi and F. Vali. The radius of the Schwarzschild black-hole in noncommutative space with GUP. Physics Procedia, 22, 2011.

[71] M. Isi, J. Mureika, and P. Nicolini. Self-completeness and generalized uncertainty principle. Journal of High Energy Physics, 2013. 
[72] S. Gangopadhyay and A. Dutta. Generalized uncertainty principle and black hole thermodynamics. General Relativity and Gravitation, 46, 2014.

[73] K. Schwarzschild. Über das gravitationsfeld eines massenpunktes nach der einsteinschen theorie. Sitzungsberichte der Deutschen Akademie der Wissenschaften zu Berlin, Klasse fur Mathematik, Physik, und Technik, page 189, 1916.

[74] K. Schwarzschild. Über das gravitationsfeld einer kugel aus inkompressibler flussigkeit nach der einsteinschen theorie. Sitzungsberichte der Deutschen Akademie der Wissenschaften zu Berlin, Klasse fur Mathematik, Physik, und Technik, page 424, 1916.

[75] A. Einstein. Näherungsweise integration der feldgleichungen der gravitation. Sitzungsberichte der Königlich Preussischen Akademie der Wissenschaften Berlin, 1916.

[76] E. G. Haug. Gravity without Newton's gravitational constant and no knowledge of mass size. preprints.org, 2018.

[77] A. T. Augousti and A. Radosz. An observation on the congruence of the escape velocity in classical mechanics and general relativity in a Schwarzschild metric. European Journal of Physics, 376:331-335, 2006. 